\title{
The 'selfish brain' is regulated by aquaporins and autophagy under nutrient deprivation
}

\author{
QIAO YE ${ }^{1,2}$, YONGHONG WU ${ }^{1}$, YAN GAO $^{1}$, ZHIHUI LI $^{1}$, WEIGUANG LI $^{1}$ and CHENGGANG ZHANG ${ }^{1}$ \\ ${ }^{1}$ Beijing Institute of Radiation Medicine, State Key Laboratory of Proteomics, \\ Cognitive and Mental Health Research Center of The People's Liberation Army, Beijing 100850; \\ ${ }^{2}$ Clinical Laboratory Center, Air Force General Hospital, PLA, Beijing 100142, P.R. China
}

Received January 12, 2015; Accepted December 23, 2015

DOI: $10.3892 / \mathrm{mmr} .2016 .4988$

\begin{abstract}
The brain maintains its mass and physiological functional capacity compared with other organs under harsh conditions such as starvation, a mechanism termed the 'selfish brain' theory. To further investigate this phenomenon, mice were examined following water and/or food deprivation. Although the body weights of the mice, the weight of the organs except the brain and blood glucose levels were significantly reduced in the absence of water and/or food, the brain weight maintained its original state. Furthermore, no significant differences in the water content of the brain or its energy balance were observed when the mice were subjected to water and/or food deprivation. To further investigate the mechanism underlying the brain maintenance of water and substance homeostasis, the expression levels of aquaporins (AQPs) and autophagy-specific protein long-chain protein 3 (LC3) were examined. During the process of water and food deprivation, no significant differences in the transcriptional levels of AQPs were observed. However, autophagy activity levels were initially stimulated, then suppressed in a time-dependent manner. LC3 and AQPs have important roles for the survival of the brain under conditions of food and water deprivation, which provided further understanding of the mechanism underlying the 'selfish brain' phenomenon. Although not involved in the energy regulation of the 'selfish brain', AQPs were observed to have important roles in water and food deprivation, specifically with regards to the control of water content. Additionally, the brain exhibits an 'unselfish strategy' using autophagy during water and/or food deprivation. The present study furthered current understanding of the 'selfish brain' theory, and identified additional regulating target genes
\end{abstract}

Correspondence to: Professor Chenggang Zhang, Beijing Institute of Radiation Medicine, State Key Laboratory of Proteomics, Cognitive and Mental Health Research Center of The People's Liberation Army, 27 Taiping Road, Beijing 100850, P.R. China

E-mail: zhangcg@bmi.ac.cn

Key words: selfish brain, aquaporin-1, aquaporin-4, autophagy of AQPs and autophagy, with the aim of providing a basis for the prevention of nutrient shortage in humans and animals.

\section{Introduction}

The brain is one of the body's most important organs, and as such has both a specific role and occupies a primary position in the organism (1). The brain is of high energy consumption $(2,3)$ and low energy capacity $(4,5)$. In addition, the brain also exhibits substrate specificity with the preference of lactate, ketones and glucose (6,7). All of the above features contribute to the mechanisms underlying energy absorption and utilization. Numerous studies investigated the mechanism underlying the brain's ability for selfishness, the combined results of which formed the 'selfish brain' theory (8-12). As previous results have demonstrated, in order to satisfy its energy requirements, the brain prioritizes the adjustment of its own ATP concentration when regulating the allocation of energy from food sources (9). The brain activates its stress system to compete for energy resources with other organs (allocation), and alters appetite (food intake) in order to alleviate the stress system and return to a state of rest (10). Under conditions of stress or nutrient deficiency, the brain safeguards its own energy supply even if this requires sacrificing the energy requirements of other organs.

Two basic hypotheses have been proposed to explain how the brain uses its regulatory methods to compete with other organs under harsh conditions: The 'lipostatic' theory and the 'glucostatic' theory. The former was originally proposed by Kennedy (11), and proposed that the brain relied on the leptin hormones in fat and muscle tissue as feedback signals (12-14). The latter proposed that blood glucose levels were used as an indicator, an important factor in the central regulatory system (15), and the brain's so called 'selfishness' would therefore be based on cerebral insulin suppression $(10,16)$. However, the adequate supply of energy to the brain is the result of both lipid conversion and the continuous supply of glucose, which combine elements of both the glucose and the lipostatic theory. Previous studies on the brain revealed that the mechanism underlying the physiological regulation and feedback signaling pathways of energy predominantly focus on absorbing energy from peripheral neurons and other organs $(8,9)$. Whether the brain uses its own substances as part of its energy supply 
source, and the role of water content in the maintenance of brain mass remain to be fully elucidated. Alterations in water content and the mechanisms underlying its regulation may further clarify the 'selfish brain' theory. The present study aimed to investigate the self-regulation of the brain under food and/or water shortage by examining autophagy and water control by AQPs. AQPs have a well-established role in water balance (17), and 6 of the 13 AQP family members have been identified in the brain. AQP-1 and 4 were observed to be the most representative proteins in the regulation of brain water content, and were demonstrated to be associated with cerebral edema (18-20). In addition, energy regulation and autophagy were also important for the brain to acquire sufficient energy. In the present study, LC3 was introduced as a protein marker to evaluate brain autophagy (21). LC3I can be phosphorylated to LC3II during autophagy. Therefore, brain autophagy is reflected by LC3II/LC3I (22). The results of the present study may further the understanding of the 'selfish brain' theory. In addition, it may provide strategies against nutrient deficiency in humans and animals, predominantly based on the target genes of AQPs and autophagy.

\section{Materials and methods}

Animal grouping and tissue sample preparation. A total of 195 female Balb/c mice (18-20 g, 4-weeks old) were provided by Vital River Laboratories Co., Ltd. (Beijing, China). All the mice were raised in the Experimental Animal Center of Beijing Institute of Radiation Medicine (Beijing, China) and maintained in specific pathogen free grade animal facility. All experiments were performed between 08:00 and 15:00 h. A maximum of five mice were housed in one cage during an experiment. The feeding room was maintained at a constant temperature of $25^{\circ} \mathrm{C}$ with normal ventilation and a natural light/dark cycle. The mice were randomly divided into four primary groups: A control group $(n=15)$, a water-deprived group $(n=60)$, a food-deprived group $(n=60)$ and a water and food-deprived group $(n=60)$. Then animals in each experimental primary group (except the control group) were the further divided into four secondary groups $(n=15)$ with deprivation durations of 1, 2, 3, 4 days. Among the mice of each secondary group, five were used for histological observation, five for the water content assay, and the remaining five mice were used for RNA and protein detection (Fig. 1). However, prior to dissection, all mice were sacrificed by cervical dislocation. Mice in each secondary group were dissected and the brain was harvested. The brain tissue samples ( 5 brains from each subgroup) were washed with normal saline and then fixed in formalin (Sinopharm Chemical Reagent Co. Ltd, Beijing, China) prior to hematoxylin and eosin (H\&E) staining. The remaining 10 brains were frozen at $-80^{\circ} \mathrm{C}$ for RNA and protein extraction.

All animal experiments were conducted in accordance with guidance for the use of experimental animals following approval by the Committee of Animal Care and Use Committee of Beijing Institute of Radiation Medicine (Beijing, China).

Body weight and blood glucose detection. Mice were weighed everyday during the experiment. The data were collected to construct a growth curve. In addition, blood glucose concentrations were detected each day as basic physiological indexes among the three experimental groups. Blood glucose was measured using blood glucose test strips (Roche Diagnostics, Basel, Switzerland) according to the manufacturer's protocol.

Analysis of water content. Lyophilization was used to detect the water content of the brain tissue samples. The tissue samples of the five mice from all secondary groups were pre-frozen in a $-80^{\circ} \mathrm{C}$ freezer for $12 \mathrm{~h}$, lyophilized in a $-50^{\circ} \mathrm{C}$ vacuum freeze-drying instrument (model FD-1A-50; Beijing Boyikang Laboratory Instruments Co., Ltd., Beijing, China) for $24 \mathrm{~h}$, and then weighed to calculate the water content.

$H \& E$ staining. Tissue samples were fixed in $4 \%$ formaldehyde solution ( $\mathrm{pH}$ 7.0; Sinopharm Chemical Reagent Co., Ltd.) for two days, and then processed for paraffin sectioning using a paraffin slicer (RM2235, Leica Microsystems, Inc., Buffalo Grove, IL, USA). The sections (5 $\mu \mathrm{m})$ were stained with hematoxylin (Sinopharm Chemical Reagent Co. Ltd.) for $3 \mathrm{~min}$, washed in tap water for $30 \mathrm{~min}$, and de-stained in warm water for $10 \mathrm{sec}$. The sections were then washed again in running water for $15 \mathrm{~min}$, and stained with eosin (Sinopharm Chemical Reagent Co., Ltd.) for $15 \mathrm{sec}$ prior to washing for $20 \mathrm{~min}$. The brain tissue sections were finally dehydrated using alcohol gradients, prior to xylene (Sinopharm Chemical Reagent Co. Ltd.) clearance and cover slipping. The stained sections were observed under a light microscope (DM2500, Leica Microsystems, Inc.).

RNA and protein extraction. The $-80^{\circ} \mathrm{C}$ preserved brain samples (100 mg/sample) were homogenized using a handheld grinder (Thermo Fisher Scientific, Inc., Waltham, MA, USA) for $1 \mathrm{~min}$ on ice. Total RNA from the mouse brains was extracted using Total RNA kits II (Omega Bio-Tek, Inc., Norcross, GA, USA) according to the manufacturer's protocol. Then, the extracted RNA $(1 \mu \mathrm{g})$ was reverse transcribed into cDNA using a Reverse Transcription system (Sigma-Aldrich, St. Louis, MO, USA).

Total protein of the animal tissue samples preserved at $-80^{\circ} \mathrm{C}$ was extracted using a cell lysis buffer (Applygen Technologies, Inc., Beijing, China) containing $50 \mathrm{mM}$ Tris (pH 7.4), $150 \mathrm{mM}$ $\mathrm{NaCl}, 1 \%$ Triton $\mathrm{X}-100,1 \%$ sodium deoxycholate, $0.1 \%$ SDS, $1 \mathrm{mM}$ sodium orthovanadate, $1 \mathrm{mM}$ sodium fluoride, $1 \mathrm{mM}$ EDTA and $1 \mathrm{mM}$ protease inhibitor, prior to western blotting.

Quantitative polymerase chain reaction ( $q P C R)$ analysis of $A Q P$ in the brain. qPCR was performed using an Illustra Ready-to-Go RT-qPCR kit (GE Healthcare Life Sciences, Chalfont, UK). All reactions were performed in a MasterCycler machine (Eppendorf, Hauppauge, NY, USA) under an initial denaturing step at $94^{\circ} \mathrm{C}$ for $5 \mathrm{~min}$, followed by 35 cycles of $94^{\circ} \mathrm{C}$ denaturation for $30 \mathrm{sec}, 55^{\circ} \mathrm{C}$ annealing for $30 \mathrm{sec}$, and $72^{\circ} \mathrm{C}$ extension for $1 \mathrm{~min}$. The genes of the AQP family were amplified by PCR using the following primers with $\beta$-actin as a reference gene: $\beta$-actin forward, 5 '-ATGATGCCCCCAGGG CTGTGTT-3' and reverse, 5'-TTGCTCTGGGCCTCATCA CCCA-3'; Aqp1 forward,5'-TTCTGGGTGGGGCCGTTCATT G-3' and reverse, 5'-TCTGTGAAGTCGCTGCTGCGTG-3'; and Aqp4 forward, 5'-AGGAAGCCTTCAGCAAAGCCGC-3' 


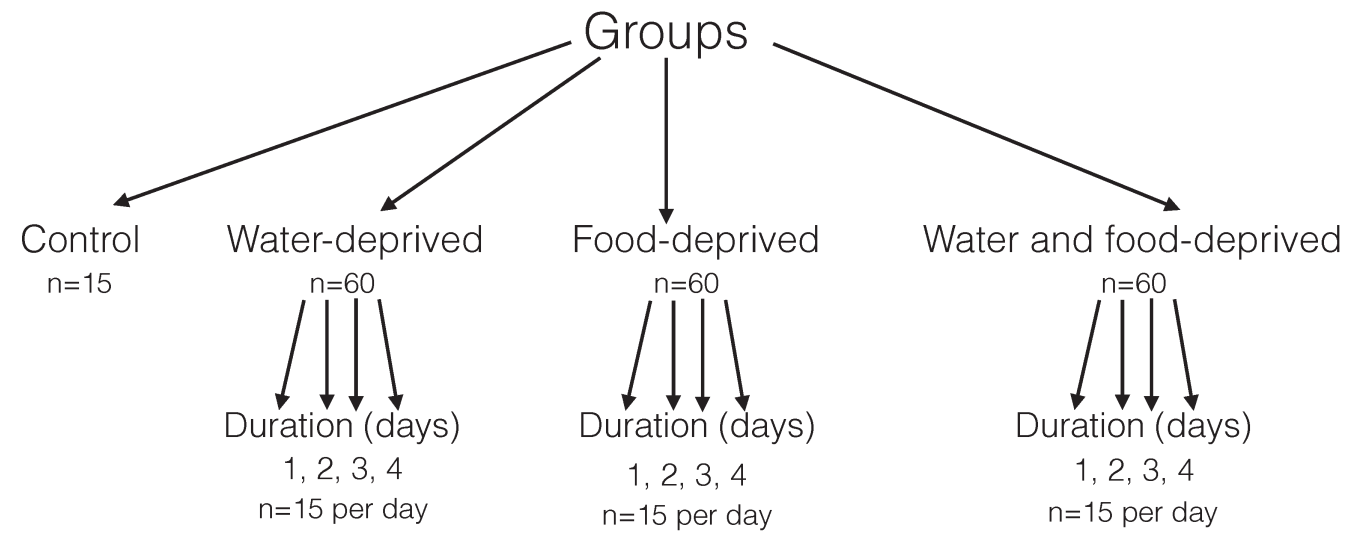

Figure 1. Schematic representation of the different treatment groups used in the current study.

A
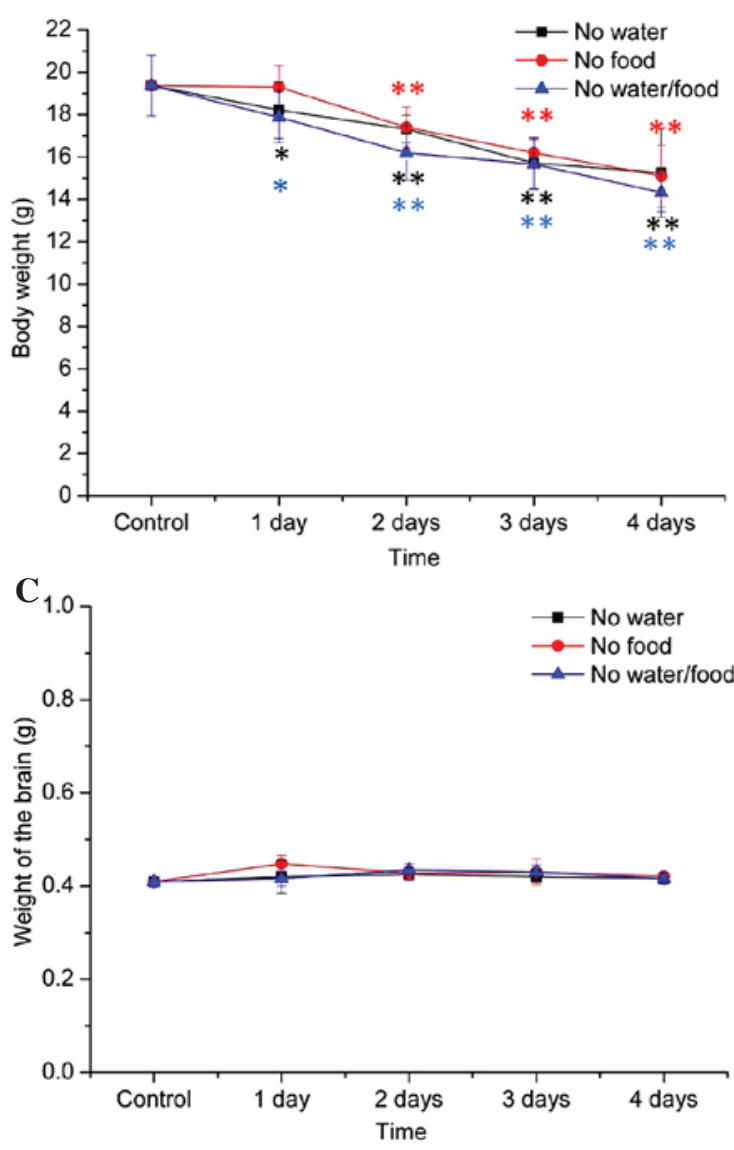

B

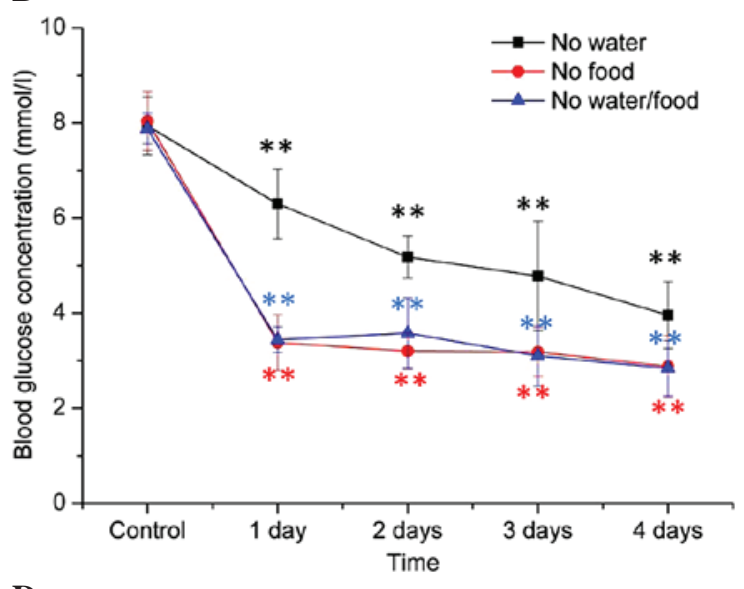

D

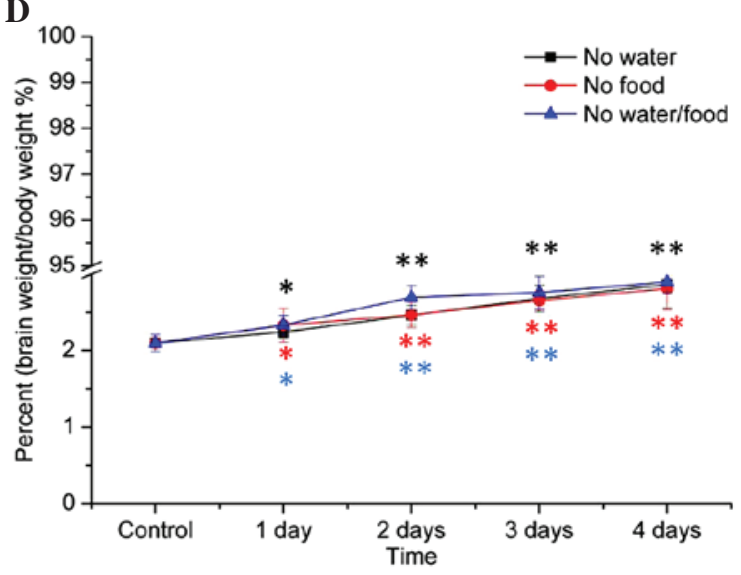

Figure 2. Mouse body weight, brain weight and blood glucose levels. (A) Body weight of the mice in the water and/or deprived groups. (B) Blood glucose concentrations of the mice during the experiment. (C) The weight of the brain in each experimental group. (D) Increasing percentage of brain weight in the whole body weight due to maintained brain weight and reducing body weight. Data are presented as the mean + standard error. ${ }^{*} \mathrm{P}<0.05{ }^{* * *} \mathrm{P}<0.01 \mathrm{vs}$ control groups.

and reverse, 5'-ACTTGGCTCCGGTTGTCCTCCA-3'. The primers were designed by the multiplex PCR primer designing web server (https://sourceforge.net/projects/mpprimer/) (23). PCR products were identified by $2 \%$ agarose gel electrophoresis (Sigma-Aldrich), and expression levels were quantified by image analysis using Image $\mathbf{J}$ for integrated optical density analysis (version 2x; National Institutes of Health, Bethesda, MA, USA), and then plotted using Origin 8.1 software (OriginLab Corporation, Northampton, MA, USA). All experiments were repeated in triplicate.
Western blot analysis of brain autophagy marker protein $L C 3 I / I I$. Total proteins (30 pg) extracted from the tissue samples from the various groups were separated by $12.5 \%$ SDS-PAGE at $60 \mathrm{~V}$ for $2.5 \mathrm{~h}$. The proteins were then transferred to a polyvinylidene difluoride membrane (PVDF; GE Healthcare Life Sciences) following the manufacturer's protocol. Then, the PVDF membranes were blocked with $5 \%$ non-fat milk (Sigma-Aldrich) for $1 \mathrm{~h}$. The membrane was probed with mouse-derived anti-LC3 primary antibody [1:2,000 in $0.1 \%$ phosphate-buffered saline with Tween 20 
No water

\begin{tabular}{|c|c|c|c|c|}
\hline Control & Day-1 & Day-2 & Day-3 & Day-4 \\
\hline \multicolumn{5}{|c|}{ No food } \\
\hline Control & Day-1 & Day-2 & Day-3 & Day-4 \\
\hline \multicolumn{5}{|c|}{ No water/food } \\
\hline
\end{tabular}
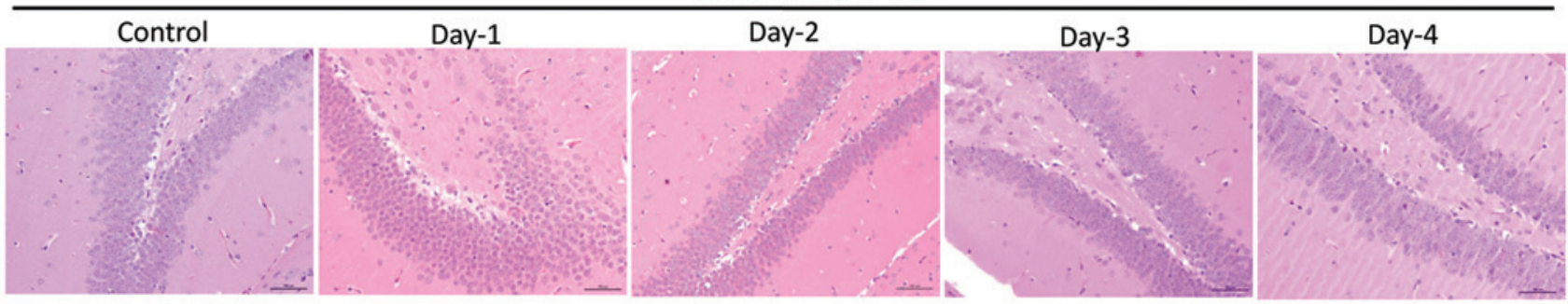

Figure 3. Hematoxylin and eosin-stained tissue sections of experimental and control murine brains. Deprivation of water and/or revealed maintained undamaged cellular structures in the mice cerebral cells under the experimental conditions. Scale bar, $50 \mu 1$; Magnification, x 200 .

(PBST; Sigma-Aldrich), and mouse-derived anti- $\beta$-actin primary antibody (1:3,000 in PBST; Sigma-Adrich)] overnight at $4^{\circ} \mathrm{C}$. The membranes were washed for $10 \mathrm{~min}, 3$ times with PBST resolution prior to incubation with horseradish peroxidase-conjugated goat anti-mouse secondary antibody (1:5,000 in PBST, Beijing Zhongshan Golden Bridge Biotechnology Co., Ltd., Beijing, China) for $1 \mathrm{~h}$ at room temperature. Chemiluminescence substrate luminal reagent (GE Healthcare Bio-Sciences) was used to detect the immunolabeled bands by exposure to X-ray films (Kodak, Rochester, NY, USA). Protein bands were also analyzed using the above-mentioned ImageJ software. All experiments were repeated at least three times.

Statistical analysis. All data are presented as mean \pm standard error. One-repeated measure analysis of two factors factorial design was using SAS (version 9.4; SAS Institure, Cary, NC, USA). One-way analysis of variance was performed in order to test for significant differences between the groups. Multiple comparisons were performed using the Student-Newman-Keuls post-hoc test. $\mathrm{P}<0.05$ was considered to indicate a statistically significant difference.

\section{Results}

Brain mass was maintained with reducing body weight and blood glucose levels. Body weight ( $\mathrm{g}$ ) and blood glucose levels $(\mathrm{mmol} / \mathrm{l})$ were measured throughout the duration of the experiment. The body weight of the mice reduced in the water and/or food deprivation groups in a time-dependent manner, and specifically in the group subjected to both food and water deprivation (Fig. 2A). The levels of blood glucose were markedly reduced on the first day of deprivation. In both the food deprivation group or the food and water deprivation group, the levels of blood glucose were rapidly reduced from the second to the fourth day. As expected, food was important for the maintenance of blood glucose levels. Mice in the water deprivation group also presented a decline in the blood glucose levels, although this reduction was not as marked as the other two groups (Fig. 2B). Conversely to body weight, the brain weight of the mice in the three primary experimental groups remained relatively stable (Fig. 2C). Therefore, the ratios of brain weight to whole body weight (\%) increased with body weight loss (Fig. 2D).

Brains of the mice are the last organ to suffer cell injury. The brains of the mice were the last organ to suffer cell injury. To examine the cell morphology of the murine tissue samples under experimental conditions, the paraffin tissue sections were stained with H\&E. As the duration of water or food shortage increased, no significant injuries in the brain structure were observed among the four groups (Fig. 3). Compared with other organs, the brain was the last organ to suffer cell injury in the body, as determined by histological observation (Fig. 3)

$A Q P$ s regulated the water content. To further explore the ability of the brain to maintain its own function under severe conditions, brain tissue samples were examined for water content at each experimental time point. Compared with the other organs, 


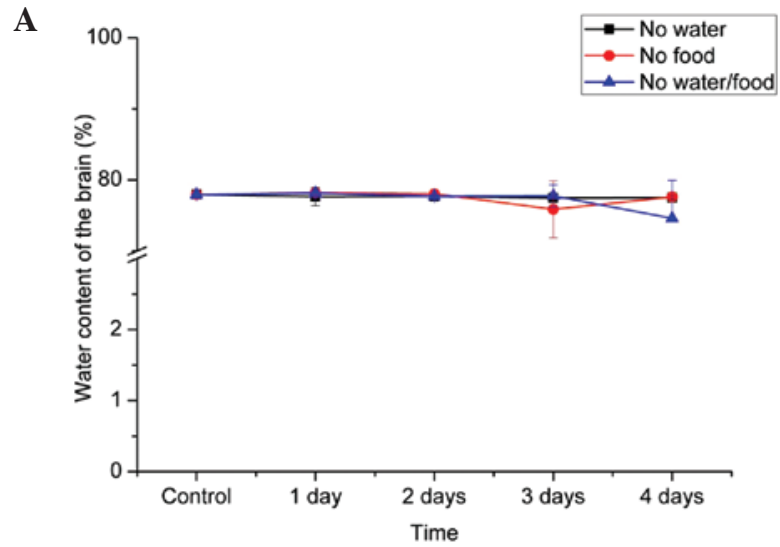

$\mathbf{B}$
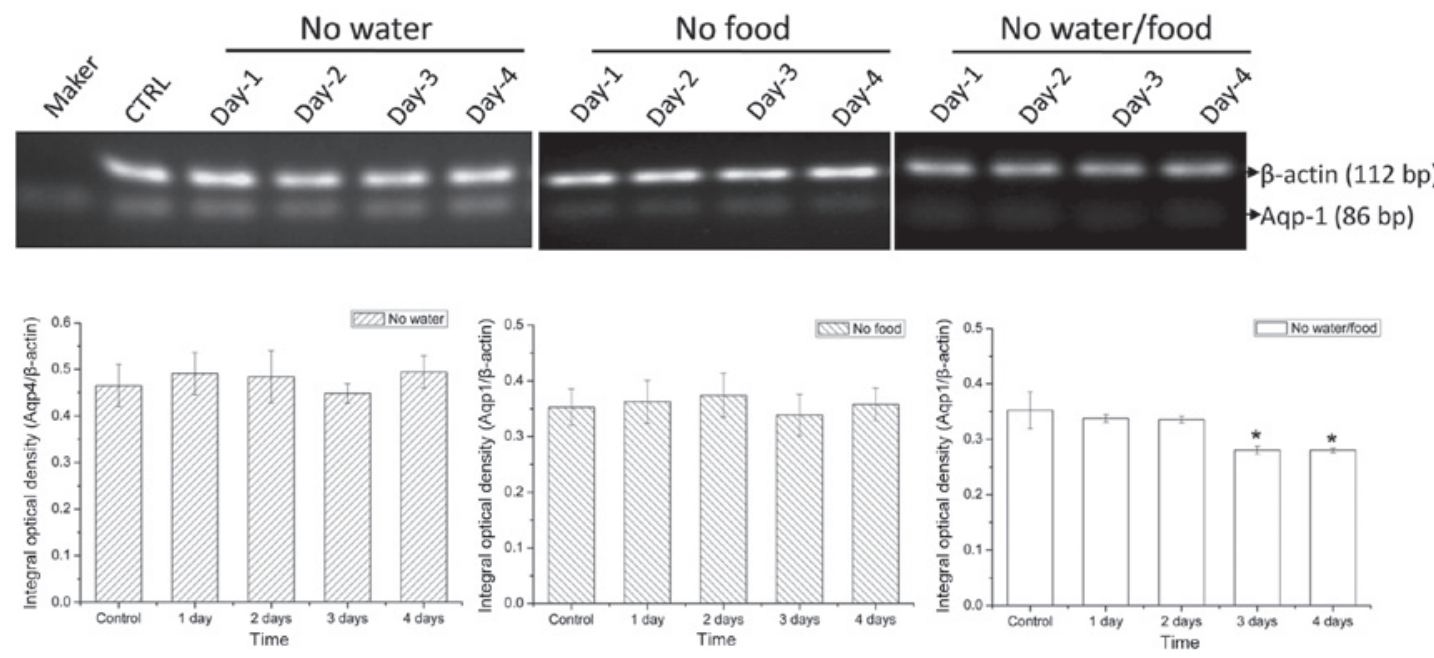

C
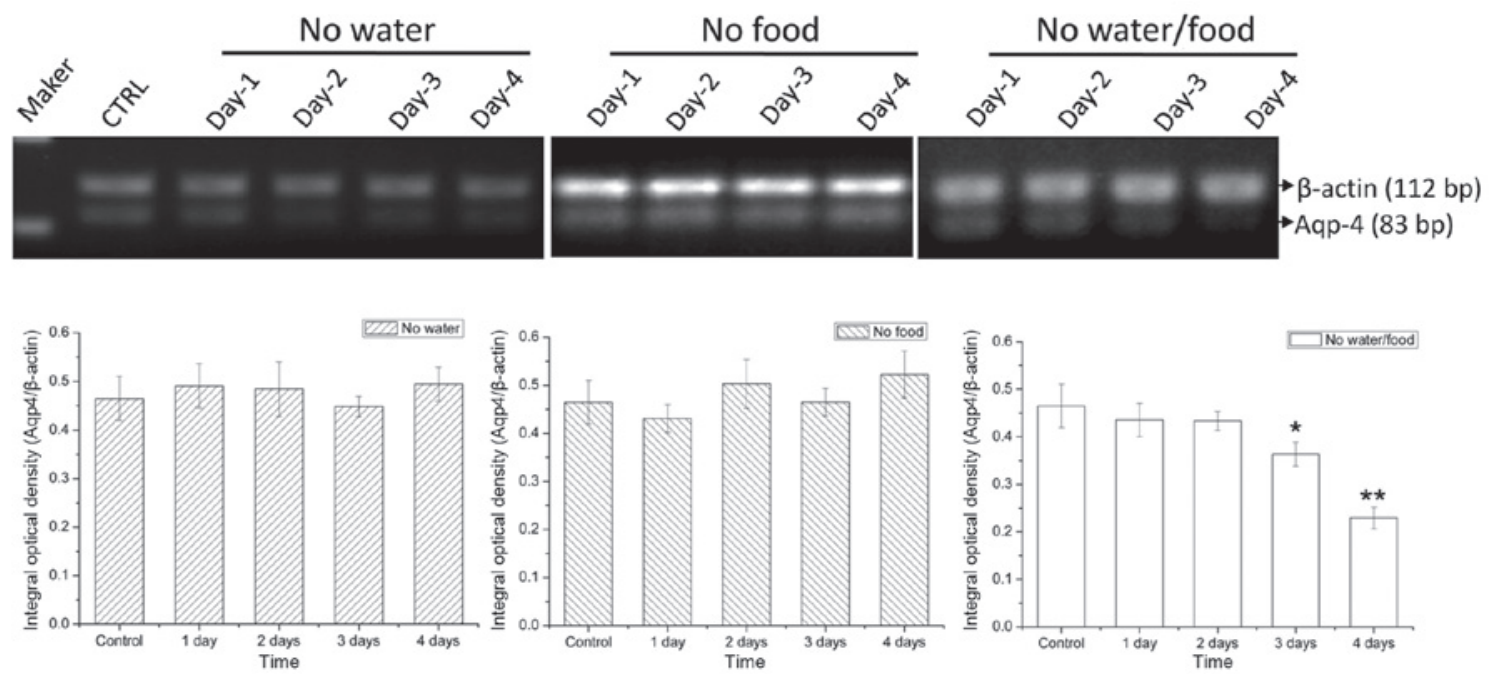

Figure 4. Water content and AQP expression levels in the brain of experimental mice. (A) Water contents of the mice brain under water or/and food deprivation. (B and C) Expression levels of AQP1 and 4 as determined by reverse transcription-quantitative polymerase chain reaction. Under water or with food deprivation, both AQP1 and 4 exhibited normal expression levels. However, under both water and food deprivation, the expression levels of AQP1 and 4 were reduced on the final two days of deprivation. Data are presented as mean + standard error. ${ }^{*} \mathrm{P}<0.05,{ }^{* *} \mathrm{P}<0.01$ vs control. The $\beta$-actin was used for internal loading control. AQP, aquaporin.

the water content of the brain (\%) showed no significant reduction (Fig. 4A), suggesting that the brain may have the ability to sequester water under harsh conditions. Furthermore, to investigate the mechanism underlying water regulation, the expression levels of two important proteins of the AQP family, AQP 1 and 4 , were examined. Compared with the control group, there were no significant alterations in AQP 1 and 4 expression levels in the water-deprived and food-deprived groups (Fig. 4B and C). However, in the water and food deprivation group, the expression levels of both AQP 1 and 4 decreased significantly on the third day of deprivation (AQP1, $\mathrm{P}=0.03671 ; \mathrm{AQP} 4, \mathrm{P}=0.02854)$ and the fourth day (AQP1, $\mathrm{P}=0.03637$; AQP4, $\mathrm{P}=0.00231$; Fig. 4B and $\mathrm{C}$ ), suggesting that the brain may have a regulatory role in maintaining water balance through AQPs. 

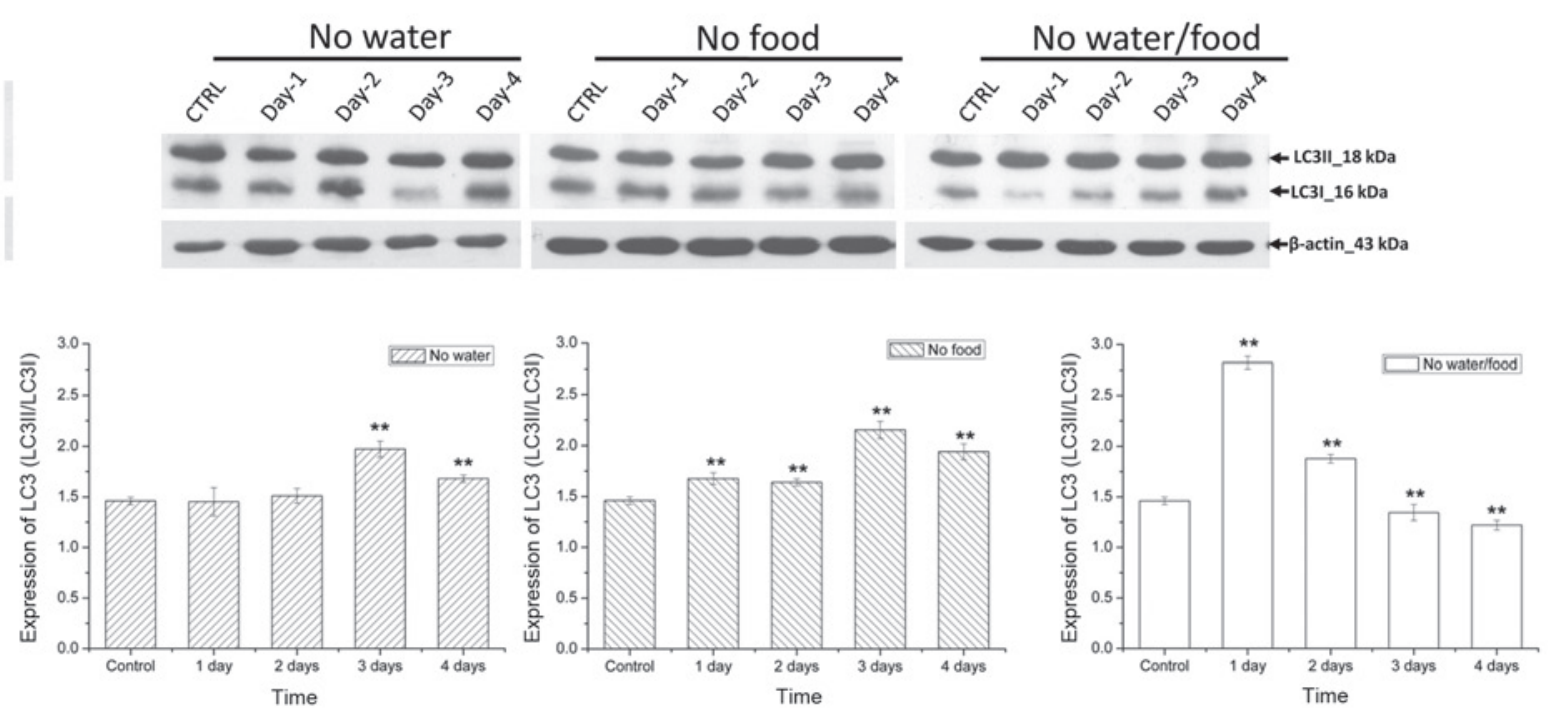

Figure 5. Western blot analysis of LC3 protein expression levels as a marker for autophagy. The relative expression levels of LC3I and LC3II that serve as markers of autophagic levels were affected more significantly by food deprivation compared with water and food and water deprivation groups, with the relative expression levels increasing on the first day of deprivation. Water deprivation also activated autophagy as the deprivation time increased, and the relative expression levels of LC3I and LC3II increased during the last two days of deprivation in the water-deprived group, and increases in autophagy in the water and food-deprived group were also observed compared with food deprivation alone. LC3, long chain protein 3.

Autophagy is involved in brain survival during lack of water and food. Energy supply was also investigated to further elucidate the mechanism underlying the ability of the brain to function under harsh conditions of deprivation. LC3II/I served as marker proteins examine the activity levels of autophagy in brain. The expression levels of LC3II/LC3I (\%) in the food deprivation group increased significantly compared with those in the control group on the first day of deprivation $(\mathrm{P}=0.00812)$, indicating that autophagy was maintained at a high level during food deprivation (Fig. 5). However, in the water deprivation group, the expression levels of LC3II/LC3I increased significantly from the third day ( $\mathrm{P}=0.00592)$. In addition, the LC3II/LC3I expression levels increased significantly from the first day in the food and water deprivation group, indicating that the activity levels of autophagy appeared early and increased under simultaneous deprivation conditions (Fig. 5).

\section{Discussion}

In the present study, mice were deprived of water and/or food. Under each experimental condition of nutritional deficiency, the body weight was reduced whereas brain weight was maintained whether under water or food shortage. These results were consistent with the 'selfish brain' theory $(10,24)$. In addition, food shortage markedly reduced blood glucose concentrations, however, water deprivation did not cause such effects. Furthermore, both water and food deprivation caused energy deficiency, which may activate the energy regulation system. Under severe energy shortage, the brain may be able to protect itself by becoming the last unaffected organ with steady water content and normal physiological structures. Therefore, it is important to elucidate the mechanism underlying the brain's unique selfish ability under harsh conditions. Considering the important role of the brain, the present study focused on water regulatory proteins and autophagy-associated proteins in the brains of mice.

In the brain, AQPs have important roles in controlling water content $(17,25)$. Six types of AQP have been revealed to be expressed in the brain, of which AQP4 was the most extensively studied, and proved to be predominantly associated with cerebral edema (18-20). To verify whether the brain mass was maintained by energy adaptation or by increasing the water content in the cerebral cells, the water content and expression levels of edema marker proteins, AQP1 and 4, were detected (26). Whether water or food-deprived, water content was not significantly affected. Therefore, cerebral edema was not present during the course of water or/and food shortage. Furthermore, compared with the control group, the expression levels of AQP1 and 4 in the group deprived of both water and food were only reduced on the third day. Alterations in AQP1 and 4 expression levels were similar, and were associated with brain water content. On the fourth day, the expression levels of both AQP1 and 4 reduced in the water and food deprivation group. In addition, brain water content was also reduced in the water and food deprivation group. Therefore, the mouse brain maintained its normal water content via regulated expression of AQP1 and 4. The brain may not only compete for energy with peripheral tissues in a selfish way, but also compete for water via AQPs.

The mice brain maintained its water supply under water shortage conditions in the same manner as energy regulation, by selfishly obtaining the energy of other organs (24). However, could the brain draw energy from its own cellular substances in an unselfish way? To answer the question, the autophagy levels in the mice brain were determined under the deprivation of water and/or food. The protein expression levels of LC3 were used as a biomarker to measure the mouse brain autophagic levels $(22,27)$. The results in the present study demonstrated that, both water and food shortage were 
able to activate autophagy in cerebral cells. Among the deprivation factors, food had an important role in activating autophagy in the early period. As previous studies noted, autophagy is the major mechanism underlying the degradation of long-lived proteins and the only known signaling pathway responsible for the degradation of organelles $(21,28)$. In addition, autophagy was regulated by nutritional status, hormonal factors and other factors including temperature, oxygen concentrations and ultraviolet radiation $(29,30)$. Autophagy is a process associated with energy re-usage in cells, and therefore food shortage may affect autophagy more directly and apparently compared with water shortage. Although the water content is less important for energy metabolism compared with food intake, long-term water shortage also caused functional disorders of the mice and the degradation of proteins in cerebral cells, which finally lead to the activation of autophagy. Therefore, in addition to competing with the energy of other organs, the cerebral cells may also use their own substance and energy resources through autophagy under harsh conditions, which complement the traditional 'selfish brain' theory (10).

In conclusion, the present study further elucidated the 'selfish brain' theory of water content maintenance via $\mathrm{AQP1}$ and 4. In addition, unlike the traditional selfish theory, the mouse brains were also demonstrated to obtain energy through autophagy, which may be considered as an unselfish method. AQPs as well as their regulatory signaling pathways merit further research to clarify the role of water content in the brain.

\section{Acknowledgements}

The present study was supported by grants from the General Program of the National Natural Science Foundation of China (grant nos. 31300703 and 81271206), the National Basic Research Project (program 973; grant no. 2012CB518200), the High-Technology Program of China (program 863; grant no. 2012AA022402), the National Key Technologies R\&D Program for New Drugs of China (grant no. 2012ZX09102301-016), The General Program of Basic Research Project of Science and Technology of Jiangsu Province (grant no. BK2012640), the Special Program of Science and Technology Development of Suzhou of Jiangsu Province (grant no. ZXY2012017) and the State Key Laboratory of Proteomics of China (grant nos. SKLP-K201004, SKLP-Y201105, SKLP-0201104 and SKLP-0201002).

\section{References}

1. Peters A, Pellerin L, Dallman M, Oltmanns KM, Schweiger U, Born J and Fehm HL: Causes of obesity: Looking beyond the hypothalamus. Prog Neurobiol 81: 61-88, 2007.

2. Shulman RG, Rothman DL, Behar KL and Hyder F: Energetic basis of brain activity: Implications for neuroimaging. Trends Neurosci 27: 489-495, 2004.

3. Bélanger M, Allaman I and Magistretti PJ: Brain energy metabolism: Focus on astrocyte-neuron metabolic cooperation. Cell Metab 14: 724-738, 2011.

4. Brown AM and Ransom BR: Astrocyte glycogen and brain energy metabolism. Glia 55: 1263-1271, 2007.

5. Rosenblatt F: The perceptron: A probabilistic model for information storage and organization in the brain. Psychol Rev 65 386-408, 1958.
6. Erecinska M, Cherian S and Silver IA: Energy metabolism in mammalian brain during development. Prog Neurobiol 73: 397-445, 2004

7. Ebert D, Haller RG and Walton ME: Energy contribution of octanoate to intact rat brain metabolism measured by $13 \mathrm{C}$ nuclear magnetic resonance spectroscopy. J Neurosci 23: 5928-5935, 2003.

8. Peters A, Schweiger U, Pellerin L, Hubold C, Oltmanns KM, Conrad M, Schultes B, Born J and Fehm HL: The selfish brain: Competition for energy resources. Neurosci Biobehav Rev 28: 143-180, 2004

9. Peters A, Schweiger U, Fruhwald-Schultes B, Born J and Fehm HL: The neuroendocrine control of glucose allocation. Exp Clin Endocrinol Diabetes 110: 199-211, 2002.

10. Hitze B, Hubold C, Van Dyken R, Schlichting K, Lehnert H, Entringer $\mathrm{S}$ and Peters A: How the selfish brain organizes its supply and demand. Fron Neuroenergetics 2: 7, 2010.

11. Kennedy GC: The role of depot fat in the hypothalamic control of food intake in the rat. Proc R Soc Lond B Biol Sci 140: 578-596, 1953.

12. Ahima RS, Prabakaran D, Mantzoros C, Qu D, Lowell B, Maratos-Flier E and Flier JS: Role of leptin in the neuroendocrine response to fasting. Nature 382: 250-252, 1996.

13. Lord GM, Matarese G, Howard JK, Baker RJ, Bloom SR and Lechler RI: Leptin modulates the T-cell immune response and reverses starvation-induced immunosuppression. Nature 394: 897-901, 1998.

14. Ahima RS, Saper CB, Flier JS and Elmquist JK: Leptin regulation of neuroendocrine systems. Front Neuroendocrinol 21: 263-307, 2000

15. Mayer J: Regulation of energy intake and the body weight: The glucostatic theory and the lipostatic hypothesis. Ann N Y Acad Sci 63: 15-43, 1955

16. Kubera B, Hubold C, Zug S, Wischnath H, Wilhelm I, Hallschmid M, Entringer S, Langemann D and Peters A: The brain's supply and demand in obesity. Front Neuroenergetics 4 : 4, 2012.

17. Badaut J, Fukuda AM, Jullienne A and Petry KG: Aquaporin and brain diseases. Biochim Biophys Acta 1840: 1554-1565, 2014.

18. Badaut J, Lasbennes F, Magistretti PJ and Regli L: Aquaporins in brain: Distribution, physiology and pathophysiology. J Cereb Blood Flow Metab 22: 367-378, 2002

19. Papadopoulos MC and Verkman AS: Aquaporin-4 and brain edema. Pediatr Nephrol 22: 778-784, 2007.

20. Suzuki R, Okuda M, Asai J, Nagashima G, Itokawa H, Matsunaga A, Fujimoto T and Suzuki T: Astrocytes co-express aquaporin-1,-4 and vascular endothelial growth factor in brain edema tissue associated with brain contusion. Acta Neurochir Suppl 96: 398-401, 2006.

21. Henell F, Berkenstam A, Ahlberg J and Glaumann $\mathrm{H}$ : Degradation of short-and long-lived proteins in perfused liver and in isolated autophagic vacuoles-lysosomes. Exp Mol Pathol 46: 1-14, 1987.

22. Tanida I, Minematsu-Ikeguchi N, Ueno T and Kominami E: Lysosomal turnover, but not a cellular level, of endogenous LC3 is a marker for autophagy. Autophagy 1: 84-91, 2005.

23. Shen Z, Qu W, Wang W, Lu Y, Wu Y, Li Z, Hang X, Wang X, Zhao D and Zhang C: MPprimer: A program for reliable multiplex PCR primer design. BMC Bioinformatics 11: 143, 2010.

24. Fehm HL, Kern W and Peters A: The selfish brain: Competition for energy resources. Prog Brain Res 153: 129-140, 2006.

25. Tait MJ, Saadoun S, Bell BA and Papadopoulos MC: Water movements in the brain: Role of aquaporins. Trends Neurosci 31: 37-43, 2008.

26. Griesdale DE and Honey CR: Aquaporins and brain edema. Surg Neurol 61: 418-421, 2004.

27. Kuma A, Matsui M and Mizushima N: LC3, an autophagosome marker, can be incorporated into protein aggregates independent of autophagy: Caution in the interpretation of LC3 localization. Autophagy 3: 323-328, 2007.

28. Mizushima N and Klionsky DJ: Protein turnover via autophagy: Implications for metabolism. Annu Rev Nutr 27: 19-40, 2007.

29. Shintani T and Klionsky DJ: Autophagy in health and disease: A double-edged sword. Science 306: 990-995, 2004.

30. Chen Y, McMillan-Ward E, Kong J, Israels S and Gibson S: Oxidative stress induces autophagic cell death independent of apoptosis in transformed and cancer cells. Cell Death Differ 15: 171-182, 2007. 